\title{
ARTICLE
}

\section{Axial strain enhances osteotomy repair with a concomitant increase in connexin43 expression}

\author{
Rishi R Gupta ${ }^{1}$, Hyunchul Kim ${ }^{2}$, Yu-Kwan Chan ${ }^{1}$, Carla Hebert ${ }^{1}$, Leah Gitajn ${ }^{1}$, David J Yoo ${ }^{1}$, Robert V O'Toole ${ }^{1}$, \\ Adam H Hsieh ${ }^{2}$ and Joseph P Stains ${ }^{1}$
}

The mechanical environment is known to influence fracture healing. We speculated that connexin43 (Cx43) gap junctions, which impact skeletal homeostasis, fracture healing and the osteogenic response to mechanical load, may play a role in mediating the response of the healing bone to mechanical strain. Here, we used an established rat fracture model, which uses a $2 \mathrm{~mm}$ osteotomy gap stabilized by an external fixator, to examine the impact of various cyclical axial loading protocols $(2 \%, 10 \%$, and $30 \%$ strain) on osteotomy healing. We examined the presence of Cx43 in the osteotomy-healing environment and assessed how mechanical strain modulates Cx43 expression patterns in the callus. We demonstrated that increased cyclical axial strain results in increased radiographic and histologic bone formation. In addition, we show by immunohistochemistry that Cx43 is abundantly expressed in the healing callus, with the expression most robust in samples exposed to increased cyclical axial strain. These data are consistent with the concept that an increase in Cx43 expression by mechanical load may be part of the mechanisms by which mechanical forces enhances fracture healing.

Bone Research (2015) 3, 15007; doi:10.1038/boneres.2015.7; Published online: 28 April 2015

\section{INTRODUCTION}

Fracture healing involves a complex array of both cellular and molecular events that occur in a sequential process to restore normal structure and function of bone. The rate and efficacy of repair depends on factors related to the patient (e.g., age, nutritional status, hormonal milieu) and factors resulting from the specifics of the injury (e.g., amount of energy imparted to the bone, fracture geometry and location). ${ }^{1-3}$ In addition, the mechanical environment has long been recognized to significantly influence the repair process. ${ }^{4}$ How precisely to optimize the mechanical environment in order to accelerate fracture healing has been the topic of intense study in orthopedic surgery for decades. ${ }^{5}$ More recently, attention has turned toward understanding the precise cellular mechanisms associated with osseous tissue transformation.

A central tenet of the bone field is that gap junctions may serve as "nodes" of an intercellular network to mediate the osteogenic response of bone. Connexin43 (Cx43) is abundantly expressed in bone and facilitates osteoblast and osteocyte communication, connecting these cells into a "functional syncytium." Of critical importance is the fact that in vivo experiments have indicated that the osteogenic response of bone cells to increased strain is not limited to the site where strain is applied, but it is a generalized phenomenon affecting a wider area of bone. ${ }^{5} \mathrm{Gap}$ junctions represent a possible mode of dissemination of these strain-generated signals among the cells of bone. Gap junctions are important for bone formation and acquisition of peak bone mass as well as response to numerous growth factors/hormones, including those involved in mechanical loading and fracture repair. ${ }^{6-7}$ Using a mouse closed femoral fracture model, conditional genetic deletion of Cx43 in mature osteoblasts and osteocytes results in impaired fracture healing, including diminished bone formation, remodeling and mechanical properties, indicating an important role for Cx43 in at least the osteoblast response following fracture. ${ }^{8}$

The aim of our study was to examine the impact of cyclical, axial load on fracture healing in our model. Assuming that our model confirmed that increased cyclic axial load across an osteotomy gap results in increased radiographic and histologic bone formation, we hypothesized that gap junctions will be increased in response to the

'Department of Orthopaedics, University of Maryland, Baltimore, MD, USA and ${ }^{2}$ Fischell Department of Bioengineering, University of Maryland, College Park, MD, USA

Correspondence: Joseph P Stains (jstains@umoa.umm.edu)

Received 6 January 2015; Revised 3 March 2015; Accepted 3 March 2015 
mechanical strain that produces an amplified healing response. In order to do this, we examined the presence of Cx43 within the multicellular fracture-healing environment and determined how mechanical load modulates Cx43 expression patterns.

\section{METHODS}

All procedures and protocols were reviewed and approved by the Institutional Animal Care and Use Committee (IACUC) at the University of Maryland. Fifty-three, six-month-old, male, Sprague-Dawley rats, each weighing approximately $500 \mathrm{~g}$, were used. The rats were maintained on rodent chow and water ad libitum and were caged in pairs prior to surgery. One rat was excluded due to the development of a deep infection. Ten rats were excluded due to technical failure of fixation postoperatively. Lastly, five animals were excluded from the analysis because after fixation their osteotomy distance was greater than $2.5 \mathrm{~mm}$, at which we consistently observed a complete failure of healing. A total of 37 rats remained for subsequent analyses, with 3-5 animals per group.

Small animal fracture model

Rats were anesthetized with isoflurane prior to undergoing a unilateral $2 \mathrm{~mm}$ mid-diaphyseal segmental osteotomy of the femur. The rats had a $2 \mathrm{~cm}$ incision and after blunt dissection between the hamstring and quadriceps muscles, the femur was exposed. A custom-made drill guide was then attached to the femur and four pre-cut 0.062-inch diameter partially threaded K-wires were inserted through pre-drilled bi-cortical holes in the mid-diaphysis. An external fixation device made of PEEK (a radiolucent biomaterial) was then attached to the K-wires with an offset of $6 \mathrm{~mm}$ in order to provide rigid fixation. The external fixator was stabilized by an aluminum locking plate. A 2 mm osteotomy was created in the stabilized area using a Hall Surgical Micro 100 oscillated saw (ConMed Linvatec, Utica, NY, USA) under constant saline irrigation (Figure 1A). Radiographs were taken immediately post-operatively using an analog faxitron machine (Faxitron X-ray, Lincolnshire, IL, USA) to ensure adequate fracture reduction and proper pin alignment. After surgery, the animals were housed individually and allowed activity ad libitum in the cage. Baytril (Enrofloxacin), a fluoroquinolone antibiotic (Bayer HealthCare), was given subcutaneously for prophylaxis against infection. Banamine (Flunixin Meglumine) was given to each animal for post-operative analgesia. Three times per week the animals had the pin sites on their external fixators cleaned with a diluted $2 \%$ chlorhexidine gluconate solution.
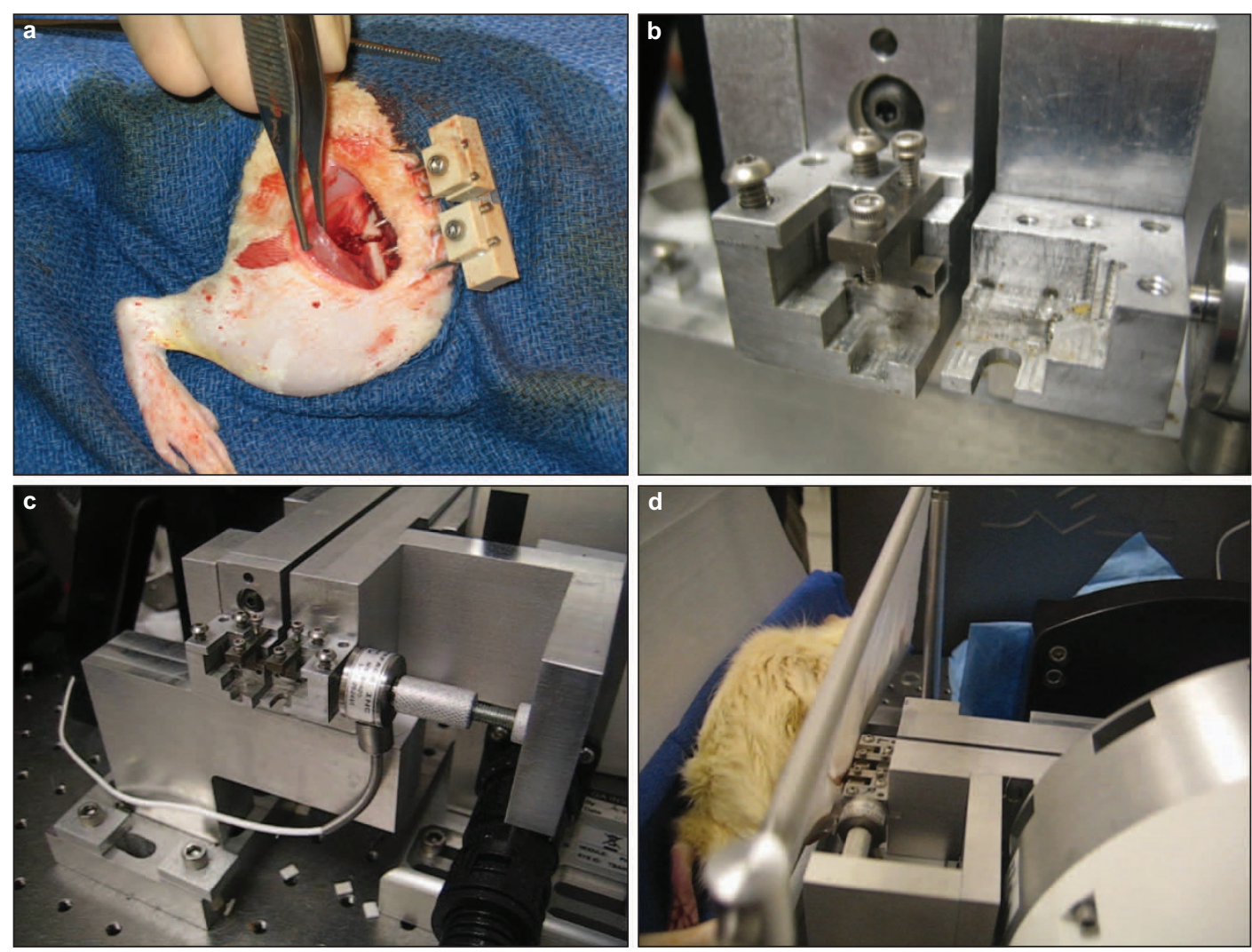

Figure 1. The osteotomy model, external fixator and axial-compression load rig. (a) Representative image showing the osteotomy, including the pin placement for the external fixation device. (b and c) Images of the load device, which interfaces with the external fixator and the material testing system to deliver cyclic axial compression across the osteotomy gap. (d) Image of a rat placed in a sling adapted to align the rat with the material testing system. 
Mechanical stimulation

Axial mechanical stimulation was performed with a custom-made fixture (Figure 1B-C) attached to a material testing system (LM-1 TestBench, Bose-Electroforce, Eden Prairie, MN, USA). Rats were anesthetized using isoflurane and the external fixators were attached to fixtures prior to removal of the locking plate (Figure 1D). Each rat was randomized to one of three strain groups $(2 \%, 10 \%$, and $30 \%)$. This micromotion was defined relative to the fracture gap (e.g., $30 \%$ strain indicated $0.6 \mathrm{~mm}$ of motion across the $2 \mathrm{~mm}$ osteotomy gap). These strains were chosen based on previous levels of strain associated with differences in healing response. ${ }^{9-10}$

Axial load was applied to the limb three times per week starting 1 week after surgery. In order to create a more uniform osteotomy-healing environment, we chose axial loading in contrast to other studies that have examined the effects of a bending moment across the osteotomy gap. Bending moments create a tensile force on one side and a compressive force on the other, and greater callus formation and better healing occurred on the compression side, ${ }^{11-12}$ which is not a difficulty experienced with axial loading. Each loading exposure was performed at $0.5 \mathrm{~Hz}$ for $17 \mathrm{~min}$ under isoflurane anesthesia using one of the three strain magnitudes $(2 \%, 10 \%$, and $30 \%)$. The frequency of loading was based on prior studies and according to Goodship and Kenwright, a frequency of loading of $0.5 \mathrm{~Hz}$ is approximately that of physiologic walking. ${ }^{13}$ In addition, 500 cycles of loading applied continuously over 17 min on each treatment day was within the range found to be most osteogenic when stimulating bone. ${ }^{13}$ The stimulation continued until 19 days post-operatively. Animals were euthanized at 10,21, and 42 days post-operatively. The 10- and 21-day time points were chosen to assess early and later effects of loading, respectively. The 42-day time point was chosen to assess whether there is a sustained response to mechanical load.

\section{Radiographic analysis}

Both femora were excised immediately following sacrifice. Soft tissue was removed from the femur while carefully preserving the fracture callus on the side with the osteotomy. Two orthogonal radiographs of both femurs were then taken using a digital faxitron machine. Three independent observers were blinded to treatment group and given a radiographic scoring system, as previously reported. ${ }^{14} \mathrm{~A}$ score of 5 indicates radiographic evidence of callus resorption after solid union. A score of 4 indicates radiographic evidence of mature callus with interfragmentary bridging. A score of 3 indicates radiographic evidence of bridging periosteal callus. A score of 2 indicates radiographic evidence of abundant callus without bridging. A score of 1 indicates radiographic evidence of little-to-moderate cal-
IUs. A score of 0 indicates no radiographic evidence of callus. Using this discrete scale, the animals at the 42-day time point were analyzed and the scores were averaged for each individual animal per treatment group.

\section{Histomorphometry and immunohistochemistry (IHC)}

Immediately post-mortem, femurs were removed, fixed in $10 \%$ neutral buffered formalin, decalcified in $14 \%$ EDTA (pH 8.0), and then embedded in paraffin. We took longitudinal cross sections through the bone and determined the depth of the cut by examining when both ends of the femoral canal were visible in the osteotomy gap. We chose this as it was a reliable means of assessing when we were centered at the osteotomy site. Serial sections were used for histologic grading, quantitative histomorphometry and IHC.

Histologic grading was done on Masson's trichrome stained sections, using a previously published scale. ${ }^{15-16}$ A score of 4 indicates complete bony union with the fracture site bridged by well-formed trabecular or cortical bone. A score of 3 indicates less than complete bony union as evidenced by the presence of a small amount of cartilage in the fracture callus. A score of 2 indicates complete cartilaginous union. A score of 1 indicates less than complete cartilaginous union with evidence of fibrous tissue in the callus. A score of 0 indicates nonunion.

Quantitative histomorphometry was performed on a minimum of three sections per sample in the area comprising the osteotomy callus, using the OsteoMeasure Bone Histomorphometry System (OsteoMetrics, Inc.) software. The following parameters were used to assess osteotomy healing and bone remodeling: cartilage volume (CV), bone volume (BV) and fibrous tissue volume (FV), each as a percentage of total volume of the callus (TV).

For $\mathrm{IHC}$, slides were incubated at $60^{\circ} \mathrm{C}$ for $1 \mathrm{~h}$ prior to deparrafinization in xylene and rehydration through a series of alcohol baths. Slides were then placed in $10 \mathrm{mM}$ sodium citrate buffer at $95^{\circ} \mathrm{C}$ for $20 \mathrm{~min}$ for antigen retrieval. A solution of $3 \%$ hydrogen peroxide in methanol was used to block endogenous peroxidase activity. An additional blocking solution (5\% normal goat serum in TBS $+0.1 \%$ Tween 20) was used prior to incubating the slides in primary antibody to rabbit anti-Cx43 (1:100 dilution; Sigma) overnight at $4^{\circ} \mathrm{C}$. After the slides were incubated in biotinylated anti-mouse secondary antibody at room temperature, the VectaStain $A B C$ reagent (Vector Labs, Burlingame, CA, USA) was used per manufacturer's directions. Slides were incubated in diaminobenzidine and counterstained with Mayer's hematoxylin. Slides underwent progressive dehydration and were coverslipped. Negative control slides were stained with non-immune rabbit IgG and secondary antibodies as above (data not shown). 
For IHC analysis, two blinded reviewers evaluated the relative amount of $\mathrm{C} \times 43$ staining in the periosteum, bone, and cartilage of the fracture callus at days 21 and 42 of healing. A scoring system was developed in order to compare the qualitative observations of $\mathrm{C} \times 43$ positive staining in the tissues/cells of the fracture callus. A score of 0 indicated that there were no cells that stained with $\mathrm{C} \times 43$ antibodies. A score of 1 indicated that there were only a few of the cells present $(<33 \%)$ that were stained. A score of 2 indicates that a moderate percentage of the cells present (33-66\%) were positively stained. A score of 3 indicates that nearly all of the cells present (66-100\%) were positively stained for $\mathrm{C} \times 43$. The results were pooled and averaged according to strain group to indicate an overall response of $\mathrm{C} \times 43$ expression to strain.

\section{Statistical analysis}

Data are presented as mean \pm standard deviations. For continuous measurements, results were compared using an analysis of variance (ANOVA) and Newman Keuls post hoc test. For discrete measurement, results were compared using an ANOVA on Ranks followed by a Bonferroni post hoc test or by chi-squared, as appropriate. A P-values less than 0.05 was interpreted as significant.

\section{RESULTS}

Radiographic and histologic evaluation

Osteotomy healing was initially assessed by analysis of plain radiographs made at the time the animals were euthanized. Radiographic analysis of mineralization in the callus at the 42-day time point demonstrated an

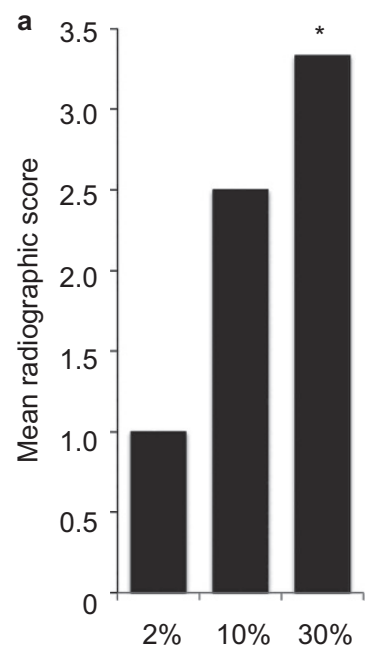

b

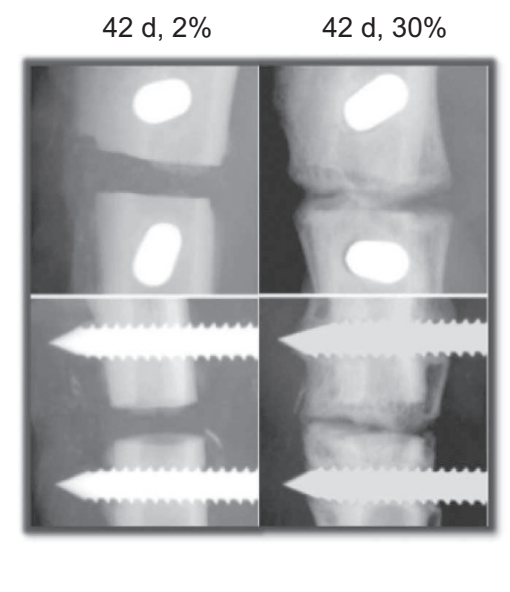

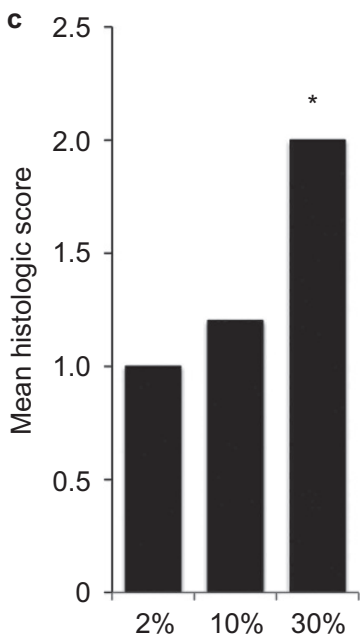
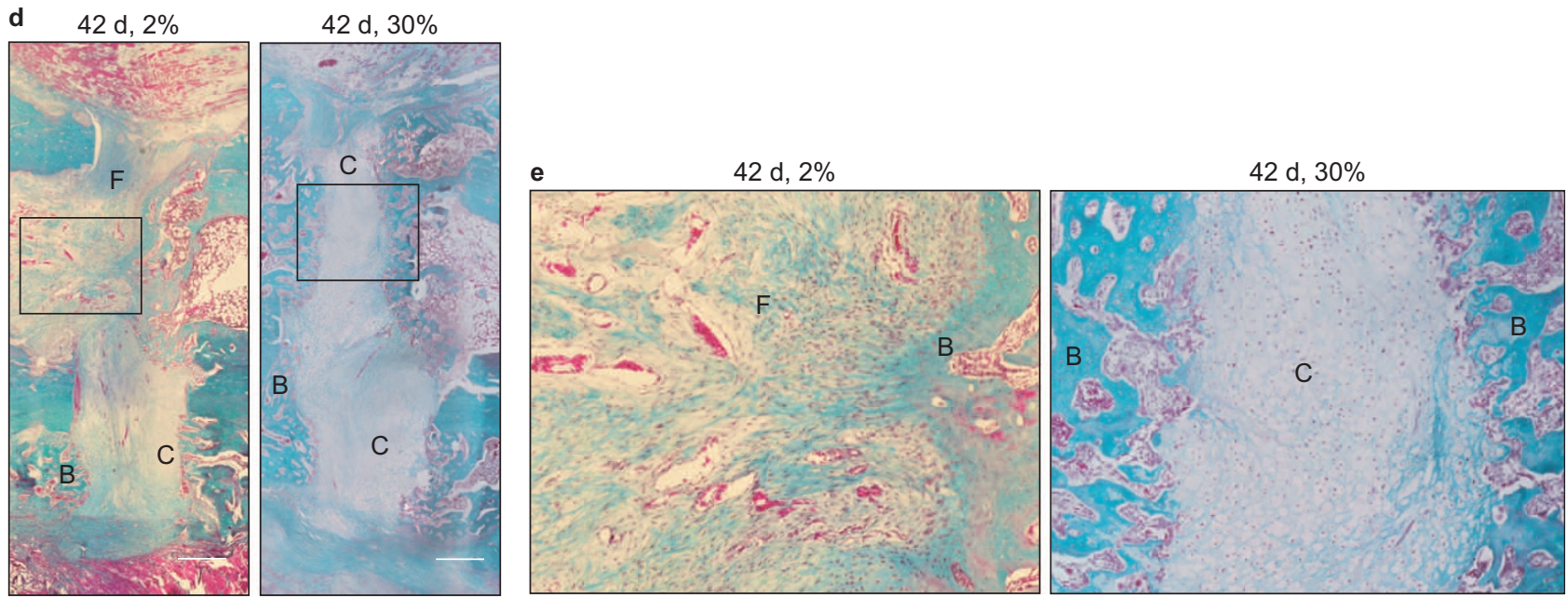

Figure 2. Radiographic and histologic analyses of mineralization in the callus at the 42-day time point. (a) Relative score for radiographic healing is shown for animals loaded at $2 \%, 10 \%$, and $30 \%$ strain at 42 -day time point $(n=3,5$, and 4 , respectively). Data shown are mean \pm SD. An * indicates a $P<0.05$ relative to the $2 \%$ strain group. (b) Representative orthogonal radiographic images from two experimental groups at 42 -day post-fracture: $2 \%$ strain (left) and 30\% strain (right). (c) Relative score for histologic healing is shown for animals loaded at 2\%, $10 \%$ and $30 \%$ strain at 42 -day time point $(n=3,5$, and 4, respectively) (d) Representative Masson's trichrome stained section of the osteotomy gap. F, fibrous tissue; C, cartilaginous tissue; B, bony tissue. The scale bar represents $0.5 \mathrm{~mm}$. The respective boxed areas are magnified in e. 
impact of the mechanical loading on the healing response. As expected, animals that were loaded at $30 \%$ strain throughout the loading protocol had a more than twofold numerical increase in radiographic and histologic osteotomy healing compared to the animals loaded at $2 \%$ strain (Figure 2). The animals in the $10 \%$ strain group at 42 days after surgery showed radiographic osteotomy healing that was intermediate to the $2 \%$ and the $30 \%$ strain groups but was not statistically different from either group. The radiographic scores generated for each of the animals were based on a continuous numerical scale. These numbers may not represent an actual increase in healing, but rather provide a means for quantitative assessment and statistical comparison in healing parameters. Thus, the twofold increase in radiographic osteotomy healing between the 2 and $30 \%$ strain groups indicates that the a

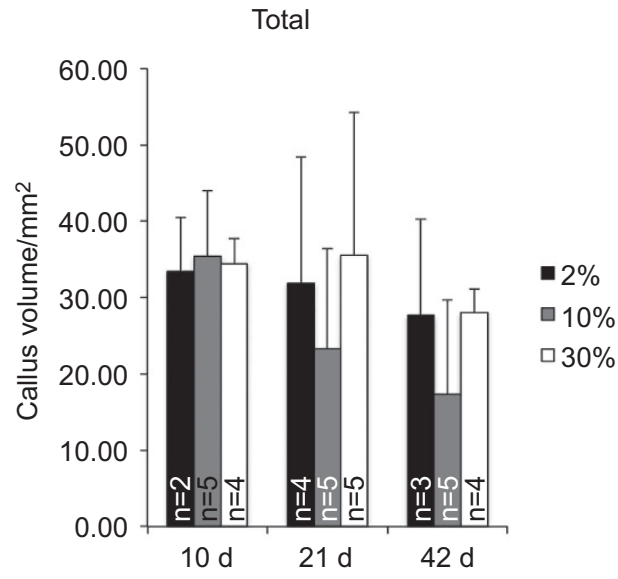

Cartilage

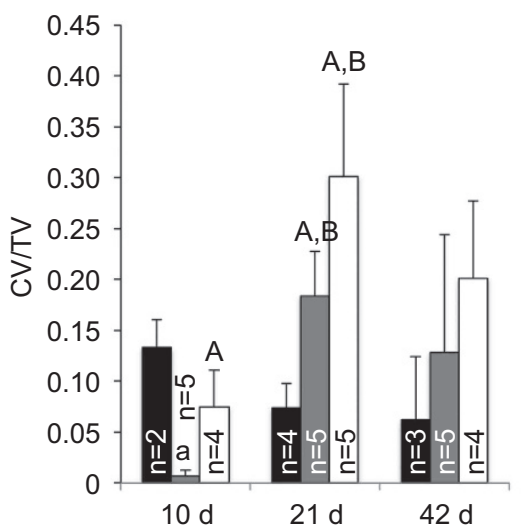

Bone

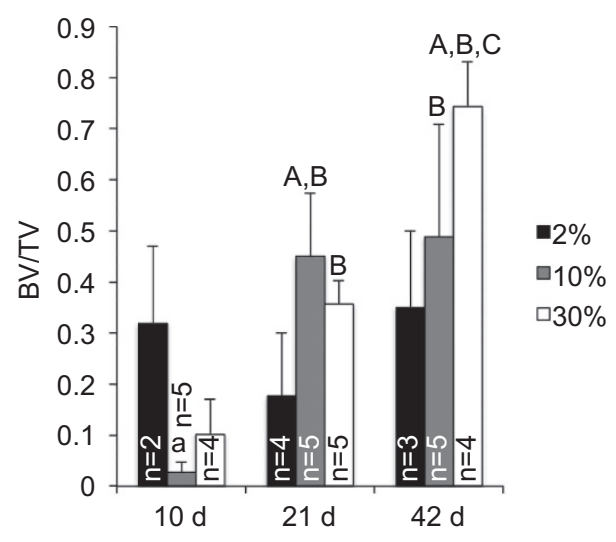

Fibrous

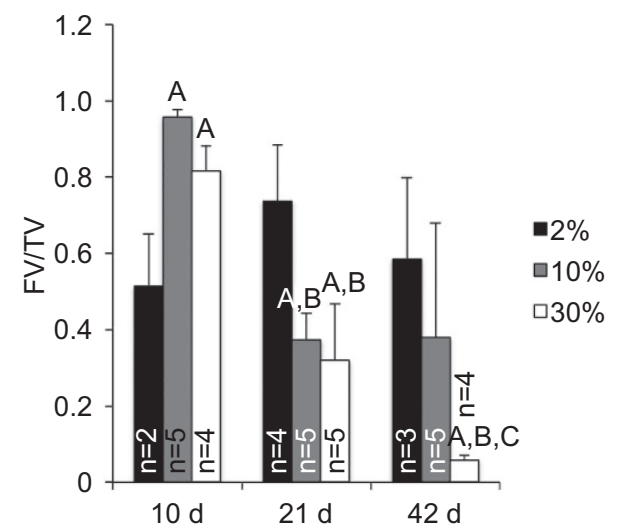

b

$2 \%$ Strain

-Bone $\square$ Cartilage $\square$ Fibrous

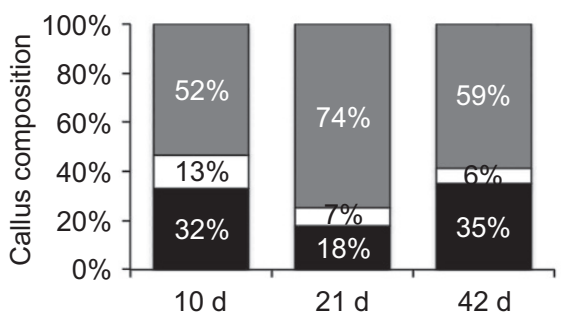

$10 \%$ Strain

-Bone $\square$ Cartilage $\square$ Fibrous

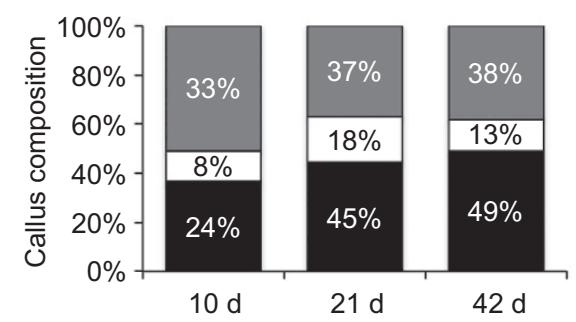

$30 \%$ Strain

घBone $\square$ Cartilage $\square$ Fibrous

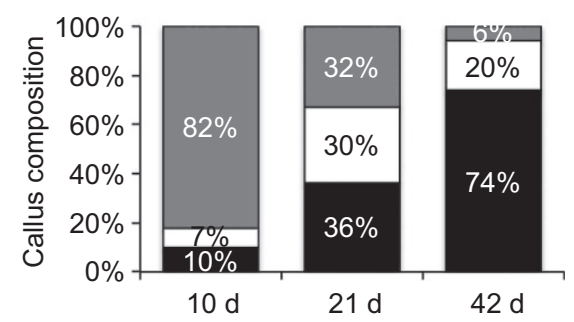

Figure 3. Histomorphometric analysis of healing in the callus. (a) Measurements for total callus volume, bone volume/total volume (BV/TV), cartilage volume/total volume (CV/TV), and fibrous volume/total volume (FV/TV) within the osteotomy callus is shown for animals loaded at $2 \%, 10 \%$, and $30 \%$ strain at the 10-, 21-, and 42-day time points. Data shown are mean $\pm \mathrm{SD}$. A indicates a $P<0.05$ relative to the $2 \%$ strain group, B indicates a $P<0.05$ relative to the 10 day, strain-matched data. C indicates a $P<0.05$ relative to the 21-day, strain-matched data. (b) Bar charts display the composition of the healing callus at each time point and for each strain group. 
loading conditions resulted in visible and measurable changes in the healing response.

\section{Histology and immunohistochemistry}

Histomorphometric analysis was performed at 10-, 21-, and 42-day post-osteotomy for animals that had been exposed to $2 \%, 10 \%$, and $30 \%$ strain (Figure 3 ). Callus volume was not statistically different at any strain or time point tested, although it trended toward a reduction in total volume at 42 days as the callus matured. At 10-day post-osteotomy, the callus composition was almost exclusively made up of fibrous tissue (FV/TV) among all the strain groups, with the exception of a single $2 \%$ animal with remarkable bony and cartilaginous callus formation at day 10 . By day 21 , the $10 \%$ and the $30 \%$ strain groups showed a marked increase in the cartilaginous (CV/TV) and bony composition (BV/TV) of the callus relative to the $2 \%$ strain group. The differences caused by cyclical axial load were most apparent at day 42 , when the callus in the $30 \%$ group was primarily composed of bony tissue, which was significantly elevated compared to the time matched $2 \%$ group. Additionally, the fibrous component of the $30 \%$ groups callus was markedly decreased relative to either the $2 \%$ or $10 \%$ strain groups.
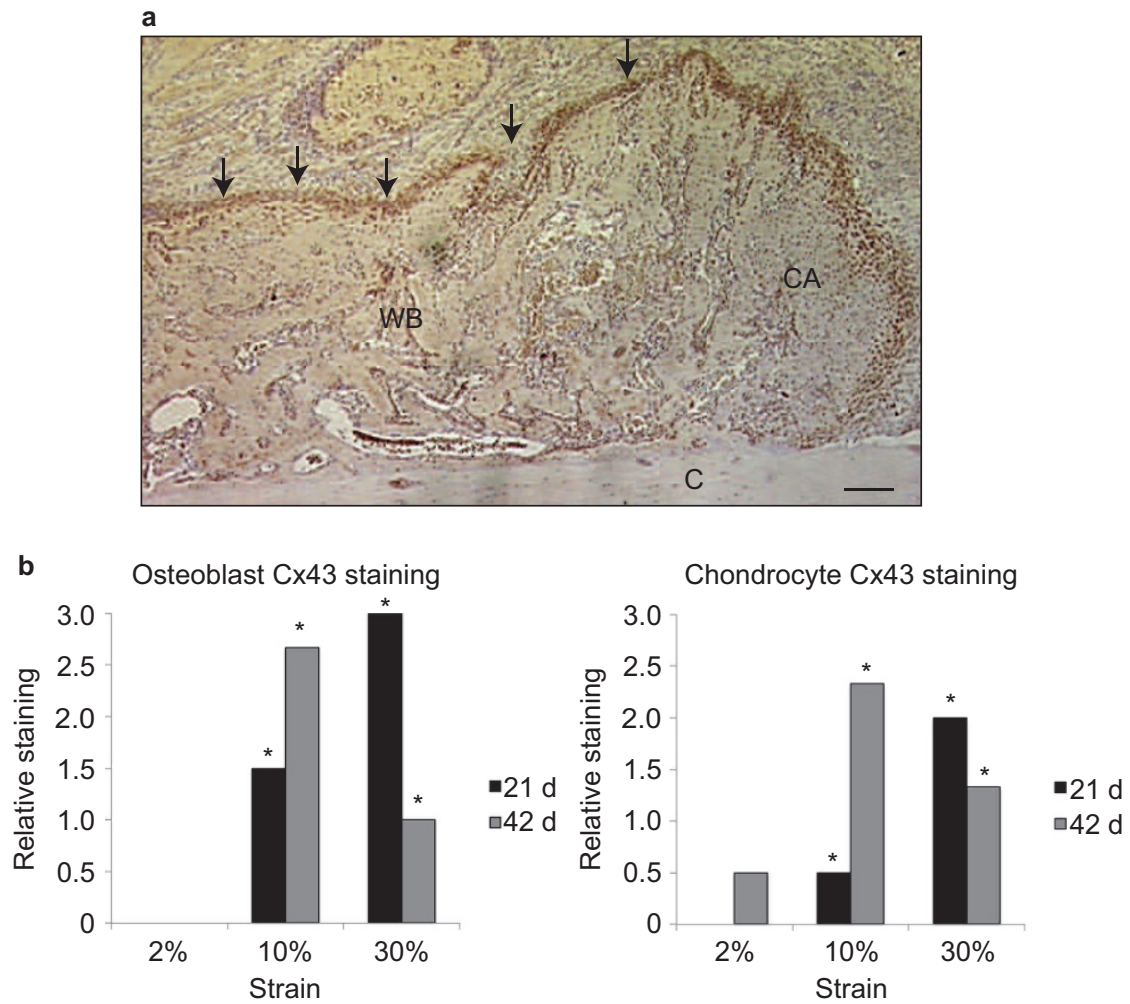

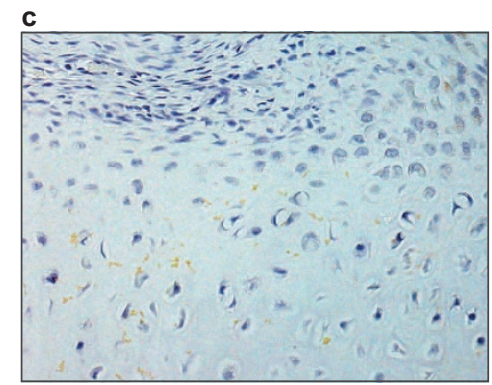

$21 \mathrm{~d}, 2 \%$

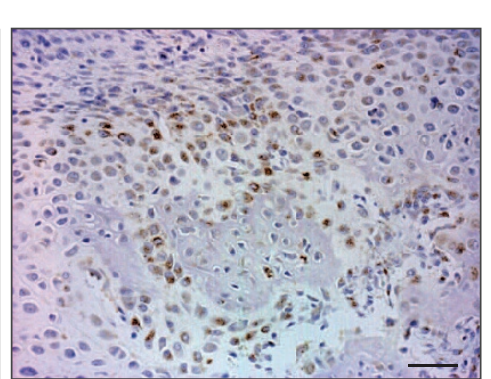

$21 \mathrm{~d}, 10 \%$

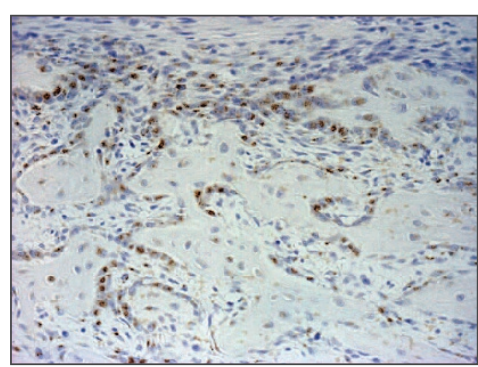

21 d, $30 \%$

Figure 4. Connexin 43 expression in the callus. (a) Representative immunostaining for Cx43 in the periosteal reaction of the callus is shown for a 10 -day, $10 \%$ strain animal. Arrows, periosteum; C, cortical bone; CA, cartilaginous tissue; WB, woven bone. Scale bar indicates $250 \mu \mathrm{m}$. (b) The mean score for the presence of $\mathrm{Cx} 43$ in the osteotomy callus is shown for animals loaded at $2 \%, 10 \%$, and $30 \%$ strain at the 21 - and 42 -day time points. An * indicates a $P$ $<0.05$ relative to the $2 \%$ strain, time point-matched data. (c) Representative immunostaining for Cx43 in the rat fracture callus is shown. Note the abundant Cx43 detected in the chondrocyte and osteoblast populations of the $10 \%$ and $30 \%$ strain group are not observed in the chondrocytes in the $2 \%$ strain group. Scale bar indicates $50 \mu \mathrm{m}$. 
In order to examine the presence of $\mathrm{Cx} 43$ in the callus and to assess the responsiveness of $\mathrm{C} \times 43$ expression to mechanical loads, IHC was completed on histologic sections of specimens from animals derived from each of the strain groups. Cx43 was robustly detected in the callus (Figure 4). Cx43 staining clearly outlined the entire fracture callus from the adjacent tissue and was present at varying levels throughout most of the cell types present in the healing callus (Figure 4A). Cx43 staining was abundant in the cells of the periosteum, bone and cartilage. The one exception was that Cx43 was consistently not detected in the fibrous tissue component of the callus. Uniformly, there was intense staining for $\mathrm{C} \times 43$ at the osteotomy site relative to distal skeletal areas, suggesting that $\mathrm{C} \times 43$ is markedly increased in the areas closer to the osteotomy site. Indeed, there was some staining for $\mathrm{C} \times 43$ that progressively tapered off further from the site of the actual osteotomy. The contralateral femurs showed considerably less evidence of Cx43 staining (lower abundance and intensity) and tended to be present in discrete packets rather than the intense, continuous staining observed in the injured bone (data not shown). In 10-day specimens, Cx43 is robustly detected in the periosteal cells, osteoblasts, and chondrocytes independent of strain group. In stark contrast to the $10 \%$ and $30 \%$ strain groups, Cx43 staining in the $2 \%$ strain group is nearly absent from the osteoblast and chondrocytes at 21 and 42 days despite the presence of these cells in the callus (Figure $4 \mathrm{~B}-\mathrm{C}$ ).

\section{DISCUSSION}

This study demonstrated that there was an impact of cyclical, axial load on osteotomy healing and further established the presence of $\mathrm{C} \times 43$ within the fracture callus. The animals in the $30 \%$ strain group showed a significant increase in the mineral content on radiographic analysis relative to the $2 \%$ strain groups. This finding was confirmed by histological analysis. Previous studies have shown that increased strain in early callus leads to greater tissue proliferation and increased callus sizes, as opposed to more rigid immobilization, which leads to an overall smaller fracture callus. ${ }^{17}$ The fracture-healing response to mechanical strain, however, is governed by complex array of factors. Timing of stimulus, gap size, number of cycles, fracture geometry, and stiffness of the construct are just some of the mechanical factors that affect the healing response..$^{5,1-12,18-20}$ Consistently, an increase in the cartilage fraction of the callus is seen with greater mechanical deformation, as was demonstrated in our study. This may, in part, be due to decreased oxygen tension as a result of microdamage to blood supply as a result of mechanical strain or it could be an intrinsic response of the mesenchymal cells to mechanical strain in the environment. ${ }^{21-23}$ An improved understanding of the biologic and mechanical factors that can impact fracture repair on the tissue level has had a significant impact on the clinical management..$^{5}$ A precise understanding of the cellular mechanisms associated with Wolff's law and osseous tissue transformation, may synergize with biomechanical principles in the development of appropriate and effective treatments for people with difficult fractures.

In addition, this is the first study to our knowledge to examine the effects of cyclical axial strain on Cx43 expression during osteotomy healing. A critical role for gap junctional communication in skeletal homeostasis and bone-forming cell function has been demonstrated both in vivo and in vitro. Data from Cx43 knockout mouse models establish that interference with $\mathrm{C} \times 43$ expression in the postnatal skeleton leads to low bone mass with an attendant defect in osteoblast function. ${ }^{24-26}$ Further, Cx43-deficient mice do not mount the same osteoanabolic response as wild-type mice to intermittent parathyroid hormone (PTH) administration ${ }^{25}$ or mechanical loads. ${ }^{27}$ Importantly, conditional deletion of Cx43 in osteoblasts has recently been shown to impair fracture healing. ${ }^{8}$ This is consistent with our data, indicating a dynamic and strain-specific regulation of $\mathrm{C} \times 43$ in the healing callus. These data suggest that $\mathrm{C} \times 43$ may facilitate some of the effects of mechanical strain on the healing tissue. Thus, it would be interesting to determine whether Cx43 deletion may also impair the mechanoresponsivness of the healing callus. Furthermore, it would be useful to determine whether the importance of $\mathrm{C} \times 43$ to fracture healing is limited to just osteoblasts and osteocytes or if it also extends to chondrocytes and periosteal cells.

Results of this study are subject to several limitations. One limitation is that the animals received Banamine during the study period, which is a non-steroidal anti-inflammatory medication. The inhibitory effects of non-steroidal medications on fracture healing are well known. ${ }^{28}$ This could partially explain the fact that there was evidence of incomplete healing at 42 days. The distribution of the anti-inflammatory agent was equal across groups and thus would not affect our intergroup comparisons; however, it may affect the results in the context of other studies. Further, the calculation for mechanical strain at the fracture site was based on pin displacement rather than an actual measurement of the relative displacement of the bone ends at the fracture site. As the strain was motioncontrolled rather than force-controlled, the actual amount of force to create the desired amount of displacement could have changed throughout the course of healing. The actual amount of displacement could also have changed slightly over time as the loading apparatus depended on the external fixator pins to create the desired movement. Despite having two pins on each side of the osteotomy to help create a more rigid construct, there could be slight variation in how much displacement occurs at the 
actual fracture site over time. As such, the actual amount of strain across the fracture site in each of the treatment groups may have varied slightly from the numbers indicated. However, the amount of strain seen between animals within treatment groups should have theoretically remained consistent.

While these limitations are important in the context of understanding the healing process at the osteotomy gap, they do not necessarily change our conclusions based on the original hypothesis. Our aim was to establish the presence of $\mathrm{Cx} 43$ in the fracture-healing environment and determine how mechanical strain modulates Cx43 expression patterns. The osteotomy healing confirmed that increased cyclic axial strain across the osteotomy gap results in increased radiographic and histologic bone formation. This allowed us to study our hypothesis that Cx43 is present and possibly modulated by varying strain.

In summary, we have demonstrated that increasing axial strain across a fracture gap results in increased bone formation both radiographically and histologically and Cx43 expression is indeed present throughout the callus. Understanding the precise cellular mechanisms associated with osseous tissue transformation and further, the limitations of host responsiveness to extracellular cues, allow for another avenue to potentially augment osteotomy healing.

\section{Competing interests}

Robert V. O'Toole is a paid consultant for Synthes, receives research support from Stryker and Synthes, and is a board member/committee appointment with the Orthoapedic Trauma Association; Adam H. Hsieh receives research support from Stryker and Synthes. Hyunchul Kim, Yu-Kwan Chan, Carla Hebert, Leah Gitajn, David J. Yoo, and Joseph P. Stains have no conflicts to report.

\section{Role of the funding source}

This research project was funded by the Department of Orthopaedics, University of Maryland (Rishi R. Gupta) and by an NIH/NIAMS grant (R01AR052719). The funding source/study sponsors had no involvement in the study design; collection, analysis and interpretation of data; the writing of the manuscript; or the decision to submit the manuscript for publication.

\section{Acknowledgements}

We would like to thank Steven Goldstein for graciously describing his surgery model, Elizabeth Smith for help with histology, Dave Cogswell for help with building the mechanical loading apparatus, Doug Powell and Shannon Hall for helping with veterinary care of our animals, and John Lowe for providing some of the materials for building the ex-fixes. This research project was funded by institutional funding by the Department of Orthopaedics, University of Maryland (Rishi R. Gupta).

\section{References}

1 Kristensen MT. Factors affecting functional prognosis of patients with hip fracture. Eur J Phys Rehabil Med 2011; 47: 257-264.

2 Ensrud KE. Epidemiology of fracture risk with advancing age. J Gerontol A Biol Sci Med Sci 2013; 68: 1236-1242.
3 Chao EY, Inoue N, Koo TK, Kim YH. Biomechanical considerations of fracture treatment and bone quality maintenance in elderly patients and patients with osteoporosis. Clin Orthop Relat Res 2004; 425: 12-25.

4 Yu X, Guo Y, Kang Q, Luo C. Effects and mechanisms of mechanical stress on secondary fracture healing. Front Biosci 2013; 18: 1344-1348.

5 Chao EY, Inoue N, Elias JJ, Aro H. Enhancement of fracture healing by mechanical and surgical intervention. Clin Orthop Relat Res 1998; 355: S163-S178.

6 Lloyd SA, Loiselle AE, Zhang Y, Donahue HJ. Shifting paradigms on the role of connexin43 in the skeletal response to mechanical load. J Bone Miner Res 2014; 29: 275-286.

7 Grimston SK, Watkins MP, Stains JP, Civitelli R. Connexin43 modulates post-natal cortical bone modeling and mechano-responsiveness. Bonekey Rep 2013; 2: 446.

8 Loiselle AE, Paul EM, Lewis GS, Donahue HJ. Osteoblast and osteocytespecific loss of Connexin 43 results in delayed bone formation and healing during murine fracture healing. J Orthop Res 2013; 31: 147-154.

9 Claes LE, Heigele CA. Magnitudes of local stress and strain along bony surfaces predict the course and type of fracture healing. J Biomech 1999; 32: 255-266.

10 Perren SM. Physical and biological aspects of fracture healing with special reference to internal fixation. Clin Orthop Relat Res 1979; 138: 175-196.

11 Smith-Adaline EA, Volkman SK, Ignelzi MA Jr et al. Mechanical environment alters tissue formation patterns during fracture repair. J Orthop Res 2004; 22: 1079-1085.

12 Hente R, Fuchtmeier B, Schlegel U et al. The influence of cyclic compression and distraction on the healing of experimental tibial fractures. J Orthop Res 2004; 22: 709-715.

13 Goodship AE, Kenwright J. The influence of induced micromovement upon the healing of experimental tibial fractures. J Bone Joint Surg $\mathrm{Br}$ 1985; 67: 650-655.

14 Chhabra A, Zijerdi D, Zhang J et al. BMP-14 deficiency inhibits long bone fracture healing: a biochemical, histologic, and radiographic assessment. J Orthop Trauma 2005; 19: 629-634.

15 Saraf SK, Singh A, Garbyal RS, Singh V. Effect of simvastatin on fracture healing-an experimental study. Indian J Exp Biol 2007; 45: 444-449.

16 Allen HL, Wase A, Bear WT. Indomethacin and aspirin: effect of nonsteroidal anti-inflammatory agents on the rate of fracture repair in the rat. Acta Orthop Scand 1980; 51: 595-600.

17 Carter DR, Blenman PR, Beaupre GS. Correlations between mechanical stress history and tissue differentiation in initial fracture healing. J Orthop Res 1988; 6: 736-748.

18 Goodship AE, Cunningham JL, Kenwright J. Strain rate and timing of stimulation in mechanical modulation of fracture healing. Clin Orthop Relat Res 1998; 355: S105-S115.

19 Goodship AE, Watkins PE, Rigby HS, Kenwright J. The role of fixator frame stiffness in the control of fracture healing. An experimental study. J Biomech 1993; 26: 1027-1035.

20 Wolf JW Jr, White AA 3rd, Panjabi MM, Southwick WO. Comparison of cyclic loading versus constant compression in the treatment of long-bone fractures in rabbits. J Bone Joint Surg Am 1981; 63: 805-810.

21 Angele P, Schumann D, Angele M et al. Cyclic, mechanical compression enhances chondrogenesis of mesenchymal progenitor cells in tissue engineering scaffolds. Biorheology 2004; 41: 335-346.

22 Gardner MJ, van der Meulen MC, Demetrakopoulos D et al. In vivo cyclic axial compression affects bone healing in the mouse tibia. J Orthop Res 2006; 24: 1679-1686.

23 Claes L, Eckert-Hubner K, Augat P. The effect of mechanical stability on local vascularization and tissue differentiation in callus healing. J Orthop Res 2002; 20: 1099-1105. 
24 Lecanda F, Warlow PM, Sheikh S et al. Connexin43 deficiency causes delayed ossification, craniofacial abnormalities, and osteoblast dysfunction. J Cell Biol 2000; 151: 931-944.

25 Chung DJ, Castro $\mathrm{CH}$, Watkins $\mathrm{M}$ et al. Low peak bone mass and attenuated anabolic response to parathyroid hormone in mice with an osteoblast-specific deletion of connexin43. J Cell Sci 2006; 119: 4187-4198.

26 Watkins M, Grimston SK, Norris JY et al. Osteoblast connexin43 modulates skeletal architecture by regulating both arms of bone remodeling. Mol Biol Cell 2011; 22: 1240-1251.

27 Grimston SK, Brodt MD, Silva MJ, Civitelli R. Attenuated response to in vivo mechanical loading in mice with conditional osteoblast ablation of the connexin43 gene (Gja1). J Bone Miner Res 2008; 23: 879886.

28 Simon AM, O'Connor JP. Dose and time-dependent effects of cyclooxygenase2 inhibition on fracture-healing. J Bone Joint Surg Am 2007; 89: 500-511.

(i) (\$) This work is licensed under a Creative Commons Attributioncc. NonCommercial-NoDerivs 3.0 Unported License. The images or other third party material in this article are included in the article's Creative Commons license, unless indicated otherwise in the credit line; if the material is not included under the Creative Commons license, users will need to obtain permission from the license holder to reproduce the material. To view a copy of this license, visit http:/ / creativecommons.org/licenses/by-nc-nd/3.0/ 